\title{
Zur Theorie der Flächen zweiten und dritten Grades.
}

(Von Herrn Geiser in Zürich.)

Durch Rotation eines Kegelschnittes um seine Hauptaxe entsteht eine Fläche zweiten Grades, welche im eigentlichen Sinne des Wortes zwei Brennpunkte besilzt und deshalb einer elementaren Behandlung zugänglich ist, die sich nicht auf andere Flächen zweiten Grades ausdehnen lässt. Man überzeugt sich in der That leicht, dass die Methoden, welche in dem ersten Bande von Jacob Steiners „Vorlesungen über synthetische Geometrie" auseinandergesetzt sind, sich sofort auf die charakterisirten Rotationsflächen ausdehnen lassen. Resultate, welche an diese Methode anknüpfen, sollen in der vorliegenden Abhandlung zunächst abgeleitet werden und weitergehenden Untersuchungen zur Einleitung dienen. -

Für die Brennpunkte einer Rotationsfläche der bezeichneten Art*) gilt der Satz: Fällt man von einem Brennpunkte aus Perpendikel auf sämmtliche Tangentialebenen der Fläche und verlängert jedes dieser Perpendikel um sich selbst, so liegen die erhaltenen Endpunkte (die Gegenpunkte des Brennpunktes in Bezug auf die Tangentialebenen) auf einer Kugel, welche den andern Brennpunkt zum Mittelpunkt und die Hauptaxe der Fläche (die gleichbedeutend mit der Hauptaxe des erzeugenden Kegelschnitles ist) zum Radius hat. Sind also von der Fläche ein Brennpunkt und eine Tangentialebene gegeben, so muss der zweite Brennpunkt der Mittelpunkt einer Kugel sein, welche durch den Gegenpunkt des ersten Brennpunktes in Bezug auf die Tangentialebene geht, und die zudem die Hauptaxe der Fläche zum Radius hat.

Man nehme ausser dem einen Brennpunkt $B$ zwei Tangentialebenen $T_{1}$ und $T_{2}$ an; der zweite Brennpunkt $A$ ist dann der Mittelpunkt einer Kugel,

*) Es giebt offenbar zwei verschiedene Arten von Rotationsłlächen zweiten Grades, die sich dadurch unterscheiden, dass bei der einen die beiden Brennpunkte reell, bei der anderen imaginär sind. Wir haben es hier zunächst mit denen der ersten Art zu thun; bei denjenigen Fragen aber, welche im Grunde algebraischer Natur sind, werden die Flächen der zweiten Art nicht von der Betrachtung ausgeschlossen werden können.

Journal fïr Mathematik Bd. LXIX. Heft 3. 
welche die Gegenpunkte $B_{1}$ und $B_{2}$ von $B$ in Bezug auf $T_{1}$ und $T_{2}$ enthält; er liegt also in der Ebene $E$, welche man in der Mitte der Strecke $B_{1} B_{2}$ senkrecht auf diese Gerade errichten kann. Diese Ebene $E$ geht auch durch die Durchschnittsgerade $g$ von $T_{1}$ und $T_{2}$; in der That steht $g$ senkrecht auf der Ebene $B B_{1} B_{2}$ und der Durchschnitt $s$ von $g$ mit dieser Ebene ist zugleich der Mittelpunkt des Kreises, welcher durch $B B_{1} B_{2}$ gelegt werden kann, womit die Behauptung erwiesen ist. Es ergiebt sich ferner: die Ebene, in welcher $A$ liegt, und die Ebene, welche durch $B$ und $g$ bestimmit ist, sind symmetrisch zu $T_{1}$ und $T_{2}$, d. h. bilden mit diesen Ebenen gleiche Winkel, ein Satz, dessen Beweis in der Ebene $B B_{1} B_{2}$ geführt wird. Man schneidet die vier Ebenen $\left(T_{1}, T_{2}\right.$, die Ebene der $A$ und die Ebene $\left.g B\right)$, welche $g$ enthalten, durch die zu $g$ senkrechte Ehene $B B_{1} B_{2}$; man hat dann in dieser Ebene vier durch $s$ gehende Geraden $s t_{1}, s t_{2}, s b, s a$, deren Ursprung durch die Bezeichnung angedeutet ist. Jetzt ist $\angle a s t_{1}=B B_{1} B_{2}=1_{2}^{1} B_{2} s B=B s t_{2}$, w. z. b. w. Damit ist auch der nachfolgende Satz evident: Legt man von der Durchschnittsgeraden zweier Tangentialebenen $T_{1}$ und $T_{2}$ einer Rotationsfläche zweiten Grades aus Ebenen durch die Brennpunkte $A$ und $B$ derselben, so sind diese Ebenen zugeordnet harmonisch zu den Halbirungsebenen der Winkel, welche $T_{1}$ und $T_{2}$ bilden. Oder auch: Sind von einer der bezeichneten Flächen ein Brennpunkt $B$ und zwei Tangentialebenen gegeben, so liegt der zweite Brennpunkt $A$ auf der Polarebene des Punktes $B$ in Bezug auf diejenige Fläche zweiten Grades, welche aus den winkelhalbirenden Ebenen von $T_{1}$ und $T_{2}$ besteht.

\section{II.}

Da nur eine einzige Kugel durch vier von einander unabhängige Punkte im Raume gelegt werden kann, so lässt sich schliessen, dass im Allgemeinen eine Rotationsfläche zweiten Grades durch einen Brennpunkt und vier Tangentialebenen vollständig bestimmt ist; der zweite Brennpunkt ist dann der Mittelpunkt derjenigen Kugel, welche durch die Gegenpunkte des gegebenen Brennpunktes in Bezug auf die vier Tangentialebenen geht. Man kann aber nach dem gefundenen Satze sagen: Legt man durch den gegebenen Brennpunkt und die Kanten des Tetraeders, welches von den Tangentialebenen gebildet wird, Ebenen und sucht zu jeder derselben eine durch die nämliche Kante gehende, mit ihr symmetrisch zu den beiden in der Kante zusammenstossenden Tetraederflächen gelegene neue Ebene, so werden die sechs auf 
diesem Wege erhaltenen Ebenen sich in dem gesuchten zweiten Brennpunkte der Fläche schneiden. Oder auch: Jede Kante des Tetraeders giebt zu einer Fläche zweiten Grades Veranlassung, die aus den winkelhalbirenden Ebenen derjenigen Tetraederflächen besteht, welche sich in ihr schneiden; sucht man nun zu dem gegebenen Brennpunkte die Polarebenen nach diesen sechs Flächen zweiten Grades, so treffen sich dieselben in dem gesuchten zweiten Brennpunkte. Die sechs erwähnten Flächen zweiten Grades haben die Mittelpunkte der acht Kugeln gemein, welche dem Tetraeder eingeschrieben werden können; diese acht Punkte liegen so, dass jede Fläche zweiten Grades, welche sieben von ihnen enthält, auch durch den achten geht. Also kann man folgende neue Form des Salzes aufstellen: Die beiden Brennpunkte eiñer Rotationsfläche zweiten Grades, welche die vier Ebenen eines Tetraeders berührt, sind conjugirte harmonische Pole in Bezug auf das Bündel der Flächen zweiten Grades, welches durch die acht Mittelpunkte der dem Tetraeder einschreibbaren Kugeln bestimmt wird. Dieses Flächenbündel ist zudem von der besondern Art, dass alle ihm zugehörigen Flächen das Tetraeder zum gemeinsamen Quadrupel haben.

III.

In Allgemeinen ist also eine Rotationsfläche zweiten Grades durch vier Tangentialebenen und einen Brennpunkt vollkommen eindeutig bestimmt, so dass zu einem ersten Brennpunkt im Allgemeinen stets ein, aber auch nur ein zweiter Brennpunkt gehört. Eine Ausnahme hiervon machen die Ecken des Fundamentaltetraeders, denn zu einer Ecke gehört als zweiter Brennpunkt jeder beliebige Punkt der gegenüberliegenden Seitenfläche; ebenso findet man, dass zu einem beliebigen Punkte, der auf einer Kante des Tetraeders liegt, alle Punkte der gegenüberliegenden Kante gehören. Man kann nämlich ein begrenztes Stück einer Geraden stets als eine Rotationsfläche zweiten Grades auffassen, deren Brennpunkte die Endpunkte der Geraden sind, und deren Tangentialebenen aus den beiden Ebenenbündeln bestehen, welche diese Endpunkte zu Millelpunkten haben. Wenn also der erste Brennpunkt eine Ebene durchläuft, so ist der Ort des zweiten eine Fläche, auf welcher die sechs Tetraederkanten liegen, die also die vier Tetraederecken zu Doppelpunkten hat, und wenn der erste Brennpunkt sich auf einer Geraden bewegt, von der vorausgesetzt wird, dass sie nicht eine der Ecken enthalte, so beschreibt der zweite Brennpunkt eine Curve, welche die vier Tetraederecken enthält. Man kann aus speciellen Fällen leicht schliessen, dass die Curve und die Fläche 
beide vom dritten Grade sind; der Nachweis, dass dies wirklich der Fall ist, wird unter Anwendung des Schlusssatzes in II. geführt, indem dann die vermutheten Sätze sich als specielle Fälle von allgemeineren darstellen, deren Erörterung von Herrn Hesse*), von Steiner **), vom Verfasser ${ }^{*}$ ) und von Herrn Sturm $+f$ ) gegeben worden ist.

Es ergiebt sich also: 1) Wenn der erste Brennpunkt einer Rotationsfläche zweiten Grades, welche die vier Seitenflächen eines Tetraeders berührt, sich auf einer, keine der Tetraederecken enthaltenden Geraden bewegt, so beschreibt der zweite Brennpunkt eine Raumcurve dritten Grades, welche durch die vier Telraederecken hindurchgeht. 2) Beschreibt der erste Brennpunkt eine Ebene, so ist der Ort des zweiten eine Fläche dritten Grades, welche die sechs Kanten des Tetraeders enthält, und welche die Ecken desselben zu Doppelpunkten hat. Auf der Fläche können leicht 28 Punkte angegeben werden. Die 28 Verbindungsgeraden der 8 Grundpunkte des Flächenbündels zweiten Grades, für welches die Punkte der Ebene $\boldsymbol{E}$ des ersten Brennpunkts und die Punkte der Fläche drilten Grades $F_{3}$ des zweiten Brennpunlits conjugirte harmonische Pole sind, schneiden $E$ in 28 Punkten, und zu jedem derselben kann man auf der ihn erzeugenden Verbindungsgeraden sofort den conjugirlen construiren.

Die einer Ebene $E$ entsprechende Fläche dritten Grades $F_{3}$ enthält ausser den Tetraederkanten noch drei Gerade, die wie folgt gefunden werden: Die Ebene $E$ schneidet das Tetraeder in einem vollständigen Vierseit, von dessen Diagonalen jede (nach unserer Zuordnung der Brennpunkte) eine Raumcurve dritlen Grades ergeben muss, die ihrer ganzen Ausdehnung nach auf $F_{3}$ liegt. Jede dieser Raumcurven aber zerfällt in drei Gerade, von denen zwei diejenigen Tetraederkanten sind, welche die zugehörige Diagonale treffen; je die drilte ist eine der gesuchten Geraden, von denen leicht nachgewiesen wird, dass sie in einer und derselben Ebene liegen. Es slossen in jeder Kante des Tetraeders zwei Seitenflächen desselben zusammen, deren Winkelhalbirungsebenen auf der Gegenkante zwei Punkte ausschneiden, so dass auf diese Weise auf jeder Tetraederkante zwei Punkte entstehen, welche von $\boldsymbol{E}$

*) „Ueber die Doppeltangenten der Curven vierter Ordnung“ Bd. 49 dieses Journ.

**) „Ueber die Flächen dritten Grades" Bd. ¿3 dieses Journ.

†) „Einige geometrische Betrachtungen" Zürcher Vierteljahrschrift Bd. X.

十†) S. dessen Werk: „Synthetische Untersuchungen über die Flächen dritter Ordnung" pag. 28 etc. 
unabhängig sind. Sucht man jetzt auf jeder Tetraederkante zu deren Durchschniltspunkt mit $E$ und den beiden vorhin bestimmten Punkten den vierten harmonischen, dem ersteren zugeordneten Punkt, so liegen die sechs neuen Punkte in einer Ebene $E^{\prime}$ als die Ecken eines vollständigen Vierseits, dessen Diagonalen sich auf $F_{3}$ befinden. Die Ebenen $E$ und $E^{\prime}$ sind offenbar reciprok, d. h. die $F_{3}^{\prime}$, welche $E^{\prime}$ entspricht, enthält drei Gerade, welche in der genannten Weise in $E$ liegen.

Eine Raumcurve dritten Grades, die durch vier feste Punkte gehen soll, ist durch zwei weitere Punkte bestimmt, und von einer Fläche dritten Grades, welche die Ecken eines gegebenen Tetraeders als Doppelpunkte enthält, können im Allgemeinen nur noch drei weitere Punkte gewählt werden. Demzufolge entspricht in der Zuordnung der Brennpunkte auch jeder Raumcurve dritten Grades durch die Ecken des Tetraeders eine Gerade und jeder Fläche dritten Grades durch die Kanten desselben eine Ebene.

\section{IV.}

Unter allen Flächen dritten Grades, welche durch die Kanten des Tetraeders gehen, ist diejenige bemerkenswerth, welche der unendlich entfernten Ebene des Raumes entspricht, und welche demzufolge der Ort der Brennpunkte aller Rotationsparaboloide ist, welche die vier Ebenen des Tetraeders berühren. Sucht man für irgend einen Brennpunkt die sämmtlichen Gegenpunkte in Bezug auf die Tangentialebenen des zugehörigen Paraboloids, so liegen dieselben auf einer Ebene, also hat die genannte specielle Fläche driller Ordnung die Eigenschaft, dass die vier Fusspunkte der Perpendikel, welche man von irgend einem ihrer Punkte aus auf die Tetraederebenen fällen kann, in derselben Ebene liegen.

Aus dieser Eigenschaft kann man umgekehrt die Brennpunktsfläche der Paraboloide herleiten, indem man einen bekannten Satz aus der Theorie der Fusspunktenflächen zu Hülfe zieht. Werden von allen Punkten des Raumes aus die Fusspunktenflächen in Bezug auf eine gegebene Fläche bestimmt und ihre Volumina berechnet, so findet sich, dass diejenigen Punkte, welche gleiche Volumina der zugehörigen Fusspunktenfläche ergeben, auf einer Fläche dritten Grades sich befinden. Als Fusspunktenfläche für ein gegebenes Telraeder in Bezug auf einen gegebenen Punkt kann man aber dasjenige neue Tetraeder bezeichnen, welches durch die vier Fusspunkte der Perpendikel gebildet wird, welche von dem angenommenen Punkte aus auf die Seitenflächen des ursprüng- 
lichen Tetraeders möglich sind. Soll der Inhalt des Fusspunktentetraeders constant und zwar gleich Null sein, so liegen seine vier Ecken in einer Ebene und umgekehrt. Dies giebt in der That den Satz, dass die Brennpunkte aller Rotationsparaboloide, welche vier gegebene Ebenen berühren, in einer Fläche dritten Grades liegen.

Auf dieser Fläche dritten Grades befinden sich die Mitten der Strecken, welche die Miltelpunkte der acht, dem Tetraeder einschreibbaren Kugeln verbinden, was aus einem in III. angegebenen allgemeinen Satze sofort folgt.

Mit der Fläche der Brennpunkte aller Rotationsparaboloide, welche vier feste Tangentialebenen gemein haben, hängt eine andere Fläche zusammen, die von den sämmtlichen Leitebenen umhüllt wird, welche diese Rotationsparaboloide zulassen. Diese Leitebenen sind die Ebenen, welche erhalten werden, wenn man von jedem Punkte der Brennpunktsfläche aus je vier Perpendikel auf die Seitenflächen des Fundamentaltetraeders fällt und durch deren Fusspunkte eine Ebene legt. Man erkennt sofort, dass jede Ebene, welche eine der vier Höhen des Tetraeders enthält, eine solche Leitebene, d. h. Tangentialebene unserer Fläche ist, ebenso wird jede Seitenfläche des Tetraeders eine Tangentialebene derselben sein. Da nun durch eine Kante des Tetraeders vier Tangentialebenen der gesuchten Fläche gehen (nämlich zwei Seitenflächen des Tetraeders und zwei Ebenen, welche die Kante und je eine der beiden, dieselbe schneidenden Höhen enthalten), aber ausser diesen keine andern, so muss die Fläche von vierter Klasse sein. Man hälte auf diese Klasse auch wie folgt schliessen können: Die vier Höhen eines Tetraeders sind vier Erzeugende derselben Schaar eines Hyperboloids, es giebt also unendlich viele Gerade, welche alle vier Höhen gleichzeitig schneiden, durch irgend eine dieser Geraden und die vier Höhen lassen sich also vier Tangentialebenen an die Fläche legen, welche demnach vierter Klasse ist.

\section{V.}

Aus dem Bisherigen folgt, dass nicht jeder beliebige Punlit des Raumes als Brennpunkt einer Rotationsfläche zweiten Grades gewählt werden kann, die fünf gegebene Ebenen berührt. Es ist also die Frage zu lösen: Welches ist der Ort der Brennpunkte aller Flächen der genannten Art, welche fünf gegebene gemeinsame Tangentialebenen haben? - Da, wie früher erwähnt, eine begrenzte Gerade stets als Rotalionsfläche zweiten Grades aufgefasst werden kann, deren Brennpunkte zugleich die Endpunkte der begrenzten Strecke 
sind, und da ferner die sämmtlichen Tangentialebenen dieser speciellen Fläche die beiden Ebenenbündel durch die Endpunkte sind, so ergiebt sich, dass auf der gesuchten Fläche der Brennpunkte die zehn Gerade liegen, in denen die gegebenen fünf Ebenen zu je zweien sich schneiden. In der That erkennt man, dass jede Ecke des Pentaeders als erster Brennpunkt einer Rotationsfläche zweiten Grades aufgefasst werden kann, deren zweiter Brennpunkt irgend ein Punkt der gegenüberliegenden Kante ist. Eine Pentaederebene enthält also vier Geraden der gesuchten Fläche, diese ist demnach mindestens vom vierten Grade.

Dass der Grad nicht höher ist, ergiebt sich aus nachfolgenden Schlüssen:

Um die Anzahl derjenigen Punkte der Fläche zu finden, welche auf einer gegebenen Geraden $g$ liegen, lassen wir das erste Mal von den fünf Pentaederebenen 1, 2, 3, 4, 5 die Ebene 5, das zweite Mal die Ebene 4 weg. Es entstehen dann zwei Tetraeder, 1234 und 1235, von denen jedes als der Ort der entsprechenden Punkte für $g$ eine Raumcurve dritten Grades ergiebt. Diese beiden Raumcurven, die im allgemeinen irreductibel sein werden, haben höchstens fünf Punkte gemein, von denen der eine, der Durchschnitlspunkt der Ebenen 1, 2, 3, als nicht von allen auftretenden Elementen abhängig, der unwesentliche Schnitt ist. Der wesentliche Schnitt besteht aus höchstens vier Punkten, welchen also höchstens vier Schniltpunlite der Geraden $g$ mit der Fläche entsprechen. Demzufolge gilt der Satz: Die Brennpunkte aller möglichen Rotationsflächen zweiten Grades, welche fünf gegebene Ebenen berühren, liegen auf einer Fläche vierten Grades, welche die zehn Kanten des Pentaeders enthält und dessen zehn Ecken zu Doppelpunkten hat ${ }^{*}$ ).

Es mag noch die Frage erledigt werden: Welches ist der Ort der Brennpunkte der sämmtlichen Rotationsflächen zweilen Grades, welche sechs gegebene Ebenen berühren? - Zunächst ist klar, dass die zwanzig Punkte, in welchen je drei der Ebenen sich schneiden, der gesuchten Curve angehören. Es seien nun die Ebenen 1, 2, 3, 4, 5, 6 gegeben, dann bilde man die Pentaeder 12345 und 12346. In jedem derselben für sich betrachtet ist die Brennpunktsfläche vom vierten Grade, die beiden schneiden sich also in einer Raumcurve sechzehnten Grades, von welcher der zu findende Ort einen Theil bilden muss. In der That treffen sich die erwähnten Brennpunktsflächen vorerst in den sechs Kanten des Tetraeders 1234, die als unwesentlicher Schnitt

*) Man bemerkt die Analogie dieser Fläche mit der namentlich durch Steiners Untersuchungen bekannt gewordenen Kernfäche einer Fläche dritter Ordnung. 
auftreten. Es bleibt jetzt als wesentlicher Schnitt der gesuchte Ort übrig, der somit eine Raumcurve zehnten Grades ist, die von jeder der funfzehn Kanten, in welchen sich die gegebenen sechs Ebenen zu je zweien schneiden, in vier Punkten getroffen wird.

Schliesslich soll noch auf das bekannte Resultat hingewiesen werden, dass es vier Rotationsflächen zweiten Grades giebt, welche sieben gegebene Ebenen berühren. -

Schwieriger ist die Erledigung derjenigen analogen Aufgaben, welche an Stelle der Tangenlialebenen Punkte selzen, so dass also Brennpunkte und Punkte als bestimmende Elemente der Rotationsflächen zweiten Grades auftreten. Die entsprechenden ebenen Probleme hat der Verfasser bereits anderswo behandelt ${ }^{*}$ ).

\section{VI.}

Die im Vorhergehenden für die Rotationsflächen zweiten Grades erhaltenen Resultate beruhen wesentlich auf den bekannten Theoremen über die conjugirten harmonischen Pole in Bezug auf ein Flächenbündel zweiten Grades. Die wenigen aus dieser Theorie nöthigen Sälze sind nicht bewiesen, sondern der in II. angegebenen Literatur entnommen worden, auf welche hier noch einmal aufmerksam gemacht werden mag. Die nachfolgenden Betrachtungen befassen sich nun näher mit der genannten Theorie und legen der Untersuchung ein vollkommen allgemeines Flächenbündel zweiten Grades zu Grunde. In Bezug auf ein solches entspricht jedem Punkte $p$ im Allgemeinen stets ein, aber auch nur ein conjugirter harmonischer Pol $p^{\prime}$, welcher der Durchschnitt der sämmtlichen Polarebenen von $p$ nach den Flächen des Bündels ist. Die Ausnahmen beschränken sich auf die Punkte einer irreductiblen Raumcurve sechsten Grades $C_{6}$ (des Ortes der Mittelpunkte der im Bündel enthaltenen Kegel), welche die Eigenschaft haben, dass jedem von ihnen nicht nur ein Pol zugehört, sondern unendlich viele Pole entsprechen, d. h. die Polarebenen eines solchen Punktes nach den sämmtlichen Flächen des Bündels schneiden sich in einer Geraden. Wenn $p$ auf einer Geraden $G$ sich bewegt, so durchläuft $p^{\prime}$ eine Raumcurve dritten Grades $C_{3}$, welche $C_{6}$ in acht Punkten

*) In seiner Inauguraldissertation: „Beiträge zur synthetischen Geometrie“. 
trifft, und wenn $p$ irgend eine Ebene $E$ beschreibt, so ist der Ort von $p^{\prime}$ eine Fläche dritten Grades $F_{3}$, auf welcher die $C_{6}$ ihrer ganzen Ausdehnung nach liegt. Die $C_{6}$ liegt auf keiner Fläche zweiten Grades, sondern sie ist theilweiser Durchschnitt zweier Flächen dritten Grades, die ausser ihr noch eine Raumcurve dritten Grades gemein haben.

Die Frage nach dem Ort der Geraden, welche den sämmtlichen Punkten der $C_{6}$ entsprechen, erledigt sich wie folgt. Da die drei Polarebenen eines Punktes $p$ der $C_{6}$ in Bezug auf drei nicht demselben Büschel des Bündels angehörigen Flächen zweiten Grades sich in einer Geraden schneiden, so kann diese im Allgemeinen gefunden werden, indem man einfach den Durchschnitt der Polarebenen von $p$ nach zweien dieser Flächen construirt. Hiebei tritt aber eine Ausnahme ein, denn das Quadrupel dieser beiden Flächen liegt auf $C_{6}$, und jeder seiner Punkte hat die Eigenschaft, dass für ihn die Polarebenen zusammenfallen. Die Polarebene eines Quadrupelpunktes nach der dritten der herausgegriffenen drei Flächen schneidet aber (wenigstens wenn das Flächenbündel allgemein ist) je eine dieser doppelten Polarebenen nur in einer Geraden und fällt nicht mit ihr zusammen. Wenn also die Aufgabe gelöst wird: „Ein Punkt beschreibt eine Raumcurve sechsten Grades, welches ist der Grad der Fläche, welche von der Durchschnittsgeraden der beiden Polarebenen jedes ihrer Punkte nach zwei Flächen zweiten Grades erzeugt wird? "6 - so wird der gefundene Grad (wegen der vier vorhin erwähnten Quadrupelebenen) um vier grösser sein als der Grad der Fläche, welche den Punkten der $C_{6}$ entspricht.

Es ist nun bekannt, dass wenn ein Punkt auf einer beliebigen Geraden $g$ sich bewegt, dann der Durchschnitt seiner Polarebenen nach zwei festen Flächen zweiten Grades ein Hyperboloid $\boldsymbol{H}_{2}$ beschreibt. Für jeden Punkt von $H_{2}$ schneiden sich die beiden Polarebenen nach den festen Flächen in einer Geraden, welche die Gerade $g$ trifft. $H_{2}$ schneidet nun eine beliebig gegebene Raumcurve $n^{\text {ten }}$ Grades in $2 n$ Punkten, also liegen auf der Fläche, welche den Punkten dieser Raumcurve entsprechen, 2n Punkte, die der Geraden angehören, d. h.: Bewegt sich ein Punkt auf einer Raumcurve $n^{\text {ten }}$ Grades, so beschreibt die jeweilige Durchschnittsgerade der Polarebenen dieses Punktes nach zwei festen Flächen zweiten Grades eine Fläche $2 n^{\text {ten }}$ Grades. Damit ist nun der Satz bewiesen: diejenigen Geraden, welche als erweiterte conjugirte Pole der Kegelmittelpunktscurve eines Flächenbündels zweiten Grades aufgefasst werden können, bilden eine geradlinige Fläche achten Grades.

Man hätte dieses Resultat noch auf anderem Wege ableiten können. Der Journal für Mathematik Bd. LXIX. Heft 3. 
Ort der conjugirten Pole aller Punkte einer Fläche $n^{\text {ten }}$ Grades in Bezug auf das vorgelegte Flächenbündel bestimmt sich leicht als eine Fläche $3 n^{\text {ten }}$ Grades. Einer Ebene $e$ entspricht eine Fläche dritten Grades $f_{3}$, der nach dem eben ausgesprochenen Satze eine Fläche neunten Grades entsprechen muss. Diese aber zerfällt in $e$ und die Fläche, welche den Punkten der $C_{6}$ entspricht, demzufolge gehört zu den Punkten der $C_{6}$ eine geradlinige Fläche achten Grades $f_{8}$, wie bereits gefunden worden.

Der schon oben erwähnte Satz, dass die einer Geraden $g$ entsprechende Raumcurve dritten Grades die $C_{6}$ in acht Punkten trifft, ergiebt sich durch diese Betrachtung voll selbst, da $g$ mit der $f_{s}$ in der That acht Punkte gemein hat, deren entsprechende auf $C_{6}$ liegen.

\section{VII.}

Durch die Theorie der conjugirten harmonischen Pole in Bezug auf ein Flächenbündel zweiten Grades wird die Abbildung der Fläche dritten Grades auf eine Ebene in der allereinfachsten Weise bewerkstelligt, und zwar folgen daraus mit Leichtigkeit die Resultate, welche Herr Clebsch ${ }^{*}$ ) mit Zugrundlegung der Grassmannschen Erzeugungsart der Flächen dritten Grades (von welcher die hier eingeschlagene vierte Steinersche Erzeugungsart ein specieller Fall ist) abgeleitet hat.

Die Ebene $E$ und die Fläche dritten Grades $F_{3}$, welche der Ort der conjugirten harmonischen Pole zu allen Punkten von $E$ in Bezug auf das Bündel ist, entsprechen sich im Allgemeinen Punkt für Punkt, und zwar so, dass ohne Ausnahme zu jedem Punkte von $F_{3}$ ein, aber nur ein Punkt auf $E$ gehört, während zwar im Allgemeinen zu jedem Punkte von $E$ ein Punkt in $F_{3}$ gehört, aber mit Ausnahmen. Die $C_{6}$ schneidet nämlich $E$ in sechs Punkten $s_{1}, \ldots s_{6}$, und jedem derselben entspricht eine Gerade, die ganz auf $F_{3}$ sich befindet. Diese Geraden werden mit $G_{1}, \ldots G_{6}$ bezeichnet, so dass dem Punkte $s_{\lambda}$ die Gerade $G_{\lambda}$ entspricht. Es soll nun zunächst gezeigt werden, dass wenn $\boldsymbol{E}$ eine ganz willkürliche Lage gegenüber dem Bündel hat und auch dieser ganz allgemein gewählt ist, dann weder die sechs Punkte $s$ auf einem Kegelschnitte, noch irgend drei von ihnen auf einer Geraden liegen können.

Lägen die Punkte $s$ für jede beliebige Ebene $E$ auf einem Kegelschnitte,

*) „Die Geometrie auf den Flächen dritter Ordnung“. Bd.65 dieses Journ. pag. 359. 
so müsste die $C_{6}$ auf einer Fläche zweiten Grades sich befinden. Wird nun eine beliebige Gerade angenommen, die keinen Punkt von $C_{6}$ enthält, so entspricht derselben, wie leicht zu beweisen, eine irreductible Raumcurve dritten Grades $C_{3}$. Durch $g$ kann man ein Ebenenbüschel legen, und diesem entspricht ein Büschel von Flächen dritten Grades, dessen Grundcurve aus $C_{6}+C_{3}$ besteht. Dieses Büschel wird von $\boldsymbol{E}$ in einem Curvenbüschel dritten Grades geschnitten, dessen Grundpunkte aus den Punkten $s$ und aus den Schnittpunkten der $C_{3}$ mit $E$ bestehen. Da nun die letztern drei nicht in einer Geraden liegen, so können die sechs ersteren sich nicht auf einem und demselben Kegelschnitte befinden.

Es liegen auch keine drei der $s$ in gerader Linie. Wäre dies der Fall, so müsste $C_{6}$ in eine Raumcurve dritten Grades $C_{3}$ und eine ebene Curve dritten Grades $C_{3}^{\prime}$ zerfallen. Sei $e$ die Ebene dieser letzteren, so wird $e$ das Flächenbündel in einem Kegelschnittnetze treffen, für welches $C_{3}^{\prime}$ die Tripelcurve ist. Da nun die Tripelcurve des Kegelschnittnetzes einen Theil der Quadrupelcurve (welche identisch mit der Kegelmittelpunktscurve ist) des Flächenbündels bildet, so lässt sich daraus schliessen, dass $e$ in Bezug auf alle Flächen des Bündels denselben Pol erzeugt, was bei einem allgemeinen Bündel, wie wir hier doch eines voraussetzen, nicht möglich ist.

Es soll ferner nachgewiesen werden, dass keine zwei der Geraden $G$ sich schneiden. Nimmt man an, dass $G_{1}$ und $G_{2}$ in $p$ sich treffen, so wird der conjugirte Pol dieses Punktes sowohl $s_{1}$ als $s_{2}$ sein müssen, d. h. die Ebene $E$ schneidet die der $C_{6}$ entsprechende geradlinige $f_{8}$ längs einer ihrer Erzeugenden, was im Allgemeinen nicht der Fall sein wird.

Schliesslich ist noch zu zeigen, dass keine vier der Geraden $G \mathbf{E r}-$ zeugende derselben Schaar eines Hyperboloides $H_{2}$ sind. Wäre dies der Fall, so müsste sich die $E$ entsprechende $F_{3}$ in $H_{2}$ und in eine Ebene $e$ spalten. Irgend einer beliebig in $E$ gelegenen Geraden, welche nicht durch einen der Punkte $s$ geht, entspricht eine irreductible Raumcurve dritten Grades, welche also ihrer ganzen Ausdehnung nach in $\mathrm{H}_{2}$ liegt, und welche nur drei Punkte mit $e$ gemein hat. Sämmtliche Punkte in $E$ mit Ausnahme der $s$ haben ihre entsprechenden auf $H_{2}$, einen Theil derselben also auf dem Kegelschnitte, der $H_{2}$ und $e$ gemein ist. Den übrigen Punkten von $e$ entsprechen aber einzig die Punkte $s$, und demzufolge müsste wenigstens einer der Punkte $s$ die Eigenschaft haben, dass seine sämmtlichen Polarebenen nach den Flächen des Bündels zusammenfallen, was beim allgemeinen Bündel ausgeschlossen ist. 
VIII.

Zu jedem der Punkte $s$ gehört eine der Geraden $G$, so dass also sämmtliche Punkte, die auf einer dieser Geraden liegen, ihre entsprechenden Punkte in dem der Geraden zugehörigen Punkte $s$ vereinigen. Wenn also eine beliebige auf $F_{3}$ verzeichnete Raumcurve $C_{n}$ die Gerade $G_{2}$ schneidet, so geht die ihr in $E$ entsprechende Curve, die, wie man weiss, höchstens vom Grade $3 n$ ist, durch $s_{\lambda}$. Wenn umgekehrt eine in $E$ gelegene irreductible Curve $C_{n}$ durch $s_{\lambda}$ geht, so entspricht ihr auf $F_{3}$ eine $C_{3 n}$, welche aber in eine $C_{3 n-1}$ und $G_{\lambda}$ zerfällt, und zwar schneiden sich diese beiden Theile. Wenn nämlich die Curve $C_{3 n-1}$ keinen Punkt mit $G_{\lambda}$ gemein hätte, so würde ihr Bild auf $E$ eine Curve sein, die nicht durch $s_{\lambda}$ geht; also könnte dieser Punkt nicht auf $C_{n}$ liegen, wie doch vorausgesetzt worden ist. Eine Curve in $E$, die $s_{\lambda}$ zum $m$ fachen Punkte hat, wird auf $F_{3}$ zu einer Raumcurve $3 m^{\text {ten }}$ Grades, welche $m$ mal die Gerade $G_{\lambda}$ enthält und sie ausserdem $m$ mal schneidet und umgekehrt. Dies ist der wesentliche Satz, auf welchen Herr Clebsch in seiner bereits citirten Abhandlung die Untersuchung der auf einer Fläche dritten Grades liegenden Raumcurven gestützt hat.

Zunächst ist es leicht, die 27 Geraden zu finden; sie werden erzeugt: 1) durch die Punkte $s$, welche die Geraden $G_{2}$ ergeben, 2) durch die sechs Kegelschnitte, die je fünf der Punkte $s$ enthalten (die Gerade auf $F_{3}$, deren entsprechender Kegelschnitt in $\boldsymbol{E}$ nicht durch $s_{\lambda}$ geht, werde mit $g_{\lambda}$ bezeichnet), 3) durch die funfzehn Geraden, die je zwei der Punkte $s$ mit einander verbinden (die Gerade auf $F_{3}$, welche der Verbindungsgeraden von $s_{\lambda}$ und $s_{\mu}$ entspricht, heisse $l_{\lambda, \mu}$ ). Es fällt nicht schwer, mit Hülfe dieser Sätze die bekannten gegenseitigen Beziehungen zwischen den einzelnen Geraden der $\boldsymbol{F}_{\mathbf{3}}$ abzuleiten, worauf aber hier nicht eingegangen werden soll. Einzig sei bemerkt, dass im Allgemeinen keine drei Geraden der Fläche dritten Grades durch denselben Punkt gehen.

Es möge noch der Abbildung der Quadrupelcurve $C_{6}$ auf $E$ erwähnt werden. Die Quadrupelcurve kann erzeugt werden als ein Theil des Durchschuittes $C_{6}+C_{3}$ der $F_{3}$ mit einer anderen Oberfläche $f_{3}$, welche einer Ebene $e$ entsprechen mag. Dieser Durchschnitt bildet sich dann in $E$ als eine Curve neunten Grades $C_{9}$ mit den $s$ als dreifachen Punkten ab, aber so, dass $C_{9}$ zerfällt und zwar in die der $C_{3}$ entsprechende Gerade $g$, welche der Durchschnitt von $e$ und $E$ ist, und in eine Curve $C_{8}$, welche sechs dreifache Punkte enthält. Da $g$ mit $C_{8}$ acht Punkte gemein hat, von denen keiner zu den Punkten 
$s$ gehört, so schliesst man, dass die $C_{3}$, welche einer beliebigen Geraden in $E$ entspricht, die $C_{6}$ in acht Punkten trifft, wie bereits in VI. bewiesen worden ist.

Für einen beliebig in $C_{8}$ gewählten Punkt liegt der conjugirte Pol auf $C_{6}$, demzufolge ist die $C_{8}$ der Durchschnitt von $E$ mit derjenigen Fläche, welche von den entsprechenden Geraden zu sämmtlichen Punkten der $C_{6}$ gebildet wird. Diese geradlinige Fläche ist also vom achten Grade; sie hat die $C_{6}$ zur dreifachen Curve und besteht aus allen denjenigen Geraden, welche die $C_{6}$ dreifach schneiden.

IX.

Die Flächen dritten Grades werden nach der Realität ihrer Geraden in Gattungen eingetheilt, deren es bekanntlich fünf giebt. Da nun die Grassmannsche Erzeugungsweise der genannten Flächen, von der die hier behandelte vierte Steinersche ein specieller Fall ist, bei reeller Annahme der bestimmenden Elemente von den fünf Gattungen nur vier giebt, so lässt sich sofort schliessen, dass sich für die vorliegende Erzeugungsweise durch eine genauere Untersuchung dasselbe Resultat ergeben muss. Diese specielle Untersuchung bietet auch neben der allgemeinern, bereits von Herrn Sturm*) durchgeführten noch ein ganz wesentliches Interesse vermöge ihrer Einfachheit.

Sie stützt sich auf die nachfolgenden Sätze: In jeder imaginären Ebene liegt eine reelle Gerade, durch jeden imaginären Punkt geht eine reelle Gerade. Irgend eine beliebige Gerade des Raumes hat entweder keinen reellen Punkt, oder einen, oder zwei (und dann unendlich viele), und demnach unterscheidet man imaginäre, punktirte und reelle Gerade. Durch jeden imaginären Punkt des Raumes lässt sich eine reelle Gerade legen, es giebt also unendlich viele reelle Geraden, welche eine imaginäre Gerade treffen. Seien $g_{1}, g_{2}, g_{3}$ drei von diesen Geraden, so werden sich keine zwei derselben begegnen, denn würden sich z. B. $g_{1}$ und $g_{2}$ treffen, so müsste $g$ in der reellen Ebene $g_{1} g_{2}$ liegen und dann einen reellen Punkt haben, was wider die Voraussetzung ist. Es folgt nun, dass jede beliebige reelle Erzeugende des Hyperboloids $g_{1} g_{2} g_{3}$, welche mit diesen Geraden derselben Schaar angehört, $g$ trifft. Es ist also leicht, durch $g$ ein reelles Hyperboloid zu legen. Uebrigens geht durch jeden reellen Punkt des Raumes ein solches Hyperboloid, da durch jeden reellen

*) A. a. O., sechstes und siebentes Kapitel. 
Punkt des Raumes eine reelle Gerade geht, welche $g$ schneidet*). (Ein reeller Punkt $p$ bestimmt nämlich mit $g$ eine Ebene, die stets eine reelle, durch $p$ gehende und $g$ schneidende Gerade enthält. Diese mit zwei anderen reellen $g$ schneidenden Geraden bestimmt das verlangte Hyperboloid.)

Wenn auf der reellen Fläche dritten Grades $F_{3}$ eine imaginäre Gerade $g$ liegt, so kann durch $g$ ein reelles Hyperboloid gelegt werden, das die $F_{3}$ ausser in $g$ noch in einer Raumcurve fünften Grades $C_{5}$ trifft, von welcher unendlich viele Punkte reell sind, da auf jeder reellen Erzeugenden von $\boldsymbol{H}_{2}$ wenigstens ein reeller Punkt von $F_{3}$ liegt. Es giebt also Ebenen, welche wenigstens drei reelle Punkte von $C_{5}$ enthalten. Eine solche schneidet aber aus $C_{5}$ noch einen reellen Punkt aus, da diese Ebene aus $F_{3}$ eine reelle Curve dritten Grades und aus $\boldsymbol{H}_{2}$ einen Kegelschnitt ausschneidet, von deren Durchschnittspunkten eine gerade Anzahl reell ist. Es giebt demnach unendlich viele Ebenen, welche die $C_{5}$ in einem imaginären und vier reellen Punkten treffen, und dies ist nicht anders möglich, als wenn $C_{5}$ in eine $C_{4}$ und eine imaginäre Gerade zerfällt. Enthält also eine reelle Fläche $F_{3}$ eine imaginäre Gerade, so ist ausser dieser einen noch eine andere vorhanden.

Durch jeden imaginären Punkt einer punktirten Geraden $g^{\prime}$ lässt sich eine reelle Gerade legen, und alle diese Geraden liegen in einer und derselben reellen Ebene. Liegt $g^{\prime}$ auf einer reellen Fläche dritten Grades $F_{3}$, so wird die durch $g^{\prime}$ mögliche reelle Ebene die $F_{3}$ in $g^{\prime}$ und in einem Kegelschnitte treffen, der die Eigenschaft hat, dass jede in seiner Ebene gelegene reelle Gerade mit ihm einen reellen und einen imaginären Punkt gemein hat. Dies ist nur dann möglich, wenn er in eine reelle und eine punktirte Gerade zerfällt, welche letztere mit $g^{\prime}$ den reellen Punkt gemein hat.

Es folgt daraus, dass, wenn auf einer reellen Fläche dritten Grades eine reelle Gerade und eine punktirte so gelegen sind, dass der reelle Punkt der letzteren auf der ersteren sich befindet, dann nothwendig drei Geraden der Fläche durch einen und denselben Punkt laufen.

X.

Wenn das Flächenbündel, in Bezug auf welches die conjugirten Pole untersucht werden, reell, d. h. durch drei, nicht demselben Büschel zugehörige

*) Die sämmtlichen reellen Geraden, welche einer imaginären Geraden begegnen, gehören einem Strahlensystem ersten Grades und erster Klasse an; durch jeden reellen Punkt geht, in jeder reellen Ebene liegt eine solche Gerade. 
reelle Flächen zweiten Grades bestimmt ist, so gehört zu jedem reellen Punkt ein reeller conjugirter $\mathrm{Pol}$, zu jeder reellen Geraden $g$ eine reelle Raumcurve dritten Grades $C_{3}$, und zu jeder reellen Ebene $E$ eine reelle Fläche dritten Grades $F_{3}$. Es folgt daraus allerdings nicht die Realität der Quadrupelcurve $C_{6}$, aber doch, dass die allfälligen imaginären Schnittpunkte der $C_{6}$ mit einer beliebigen reellen Ebene paarweise conjugirt sind. In der That, wenn man das reelle Flächenbüschel betrachtet, welches einem reellen Ebenenbüschel mit der Axe $g$ entspricht, so findet man, dass dasselbe durch eine willkürliche Ebene $E$ in einem reellen Curvenbüschel dritten Grades geschnitten wird, von dessen neun Grundpunkten wenigstens einer reell ist, während die übrigen paarweise conjugirt sind. Von diesen neun Grundpunkten fallen drei auf den Durchschnitt der reellen $g$ entsprechenden $C_{3}$ mit $E$, der entweder aus drei reellen Punkten, oder einem reellen und zwei conjugirten Punkten besteht. Die übrigen sechs Punkte, offenbar die $s$, welche $C_{6}$ mit $E$ gemein hat, sind also paarweise conjugirt, insofern sie nicht reell sind.

Einem reellen Punkte $s$ entspricht nothwendigerweise eine reelle Gerade $G$, während einem imaginären $s$ eine imaginäre $G$ zugehört, denn wäre auf derselben ein reeller Punkt vorhanden, so müsste demselben ein reelles $s$ entsprechen.

In Bezug auf die $s$ haben wir also folgende vier Fälle zu unterscheiden:

I. alle $s$ sind reell;

II. vier $s$ sind reell und zwei conjugirt imaginär;

III. zwei $s$ reell und zweimal zwei conjugirt imaginär;

IV. dreimal zwei $s$ sind conjugirt imaginär.

Jedem dieser Fälle entspricht in der That in Bezug auf die Realität der Geraden eine besondere Gattung von Flächen dritten Grades, wie vorausgesehen worden ist, und wie die genauere Betrachtung zeigen soll.

ad I. Da die $s$ alle reell sind, so sind die Verbindungsgeraden derselben zu zweien so wie auch die Kegelschnitte, welche je fünf von ihnen enthalten, reell und demzufolge alle 27 Geraden der Fläche reell.

ad II. Es seien $s_{1}, s_{2}, s_{3}, s_{4}$ reell und die beiden übrigen $s_{5}$ und $s_{6}$ conjugirt imaginär, dann sind auch $G_{1}, G_{2}, G_{3}, G_{4}$ reell und $G_{5}$ und $G_{6}$ imaginär. Von den 15 Geraden $l_{\lambda, \mu}$ sind offenbar reell: $l_{12}, l_{13}, l_{14}, l_{23}, l_{24}, l_{34}$, $l_{56}$ (letztere, weil $s_{5}$ und $s_{6}$ als conjugirte Punkte in $E$ durch eine reelle Gerade verbunden werden können). Es ist auch leicht $\mathrm{zu}$ sehen, dass diejenigen Geraden, welche den Kegelschnitten $s(56123), s(56124), s(56134), s(56234)$ 
entsprechen, reell sind, da diese Realität nur von der Realität der Kegelschnitte abhängt. Man kann aber, weil $s_{5}$ und $s_{6}$ conjugirt sind, dieselben als Doppelpunkte eines reellen Punktsystems auffassen, so dass jeder der genannten Kegelschnitte durch drei reelle Punkte und ein reelles Punktsystem bestimmt, und deshalb selber reell ist. Wäre auch der Kegelschnitt $s$ (12345) reell, so müsste derselbe durch $s_{6}$ gehen, was ausgeschlossen ist; aus demselben Grunde ist $s(12346)$ imaginär. Von den Geraden $g$ sind also $g_{1}, g_{2}, g_{3}, g_{4}$ reell, $g_{5}, g_{6}$ nicht.

Von den nicht reellen Geraden der vorliegenden Fläche $F_{3}$ kann keine einen reellen Punkt enthalten. Wäre z. B. ein reeller Punkt auf $l_{15}$ vorhanden, so läge derselbe entweder auf $G_{1}$ oder $G_{5}$ oder ausserhalb dieser beiden Geraden. Das letztere ist nicht möglich, denn einem solchen Punkte würde in $E$ ein reeller, nicht mit $s_{1}$ zusammenfallender Punkt entsprechen, der mit $s_{1}$ eine reelle Gerade $s_{1} s_{5}$ bestimmen würde, was nicht möglich ist, da sonst $s_{5}$ auf zwei reellen Geraden $s_{1} s_{5}, s_{5} s_{6}$ läge. Auf $G_{5}$ kann der angenommene reelle Punkt nicht liegen, denn $G_{5}$ enthält überhaupt keinen reellen Punkt, also wäre nur denkbar, dass $G_{1}$ und $l_{15}$ einen reellen Punkt gemein hätten. Nach dem Schlusssatze von IX. würden dann drei Geraden der Fläche durch denselben Punkt gehen, was aber im allgemeinen nicht statt findet. Aus demselben Grunde hat auch keine der andern Geraden, sei sie $l$ oder $g$, einen reellen Punkt. Die Gattung II. hat also 15 reelle und 12 imaginäre Gerade. ad III. $s_{1}$ und $s_{2}$ sind reell, $s_{3}$ und $s_{4}, s_{5}$ und $s_{6}$ conjugirt imaginär. Die Geraden $G_{1}, G_{2}$ sind reell, die übrigen $G$ imaginär. Die Kegelschnitte $s(34561)$ und $s(34562)$ sind reell, also $g_{1}$ und $g_{2}$ ebenfalls. Von den übrigen durch je fünf der sechs $s$ möglichen Kegelschnitte hat jeder nur die beiden reellen Punkte $s$ und $s_{2}$ und ist im Uebrigen imaginär, demzufolge sind auch $g_{3}, g_{4}, g_{5}, g_{6}$ imaginär. Von den 15 Verbindungsgeraden der $s$ sind reell: $s_{1} s_{2}, s_{3} s_{4}, s_{5} s_{6}$, so dass ihnen die drei reellen Geraden $l_{12}, l_{34}, l_{56}$ entsprechen. Die Geraden $l_{13}, l_{14}, l_{15}, l_{16}, l_{23}, l_{24}, l_{25}, l_{26}$ sind imaginär, weil ihre Abbildungen auf $E$ ausser resp. $s_{1}$ und $s_{2}$ keinen reellen Punkt haben. Von den Geraden $l_{35}$ und $l_{46}, l_{36}$ und $l_{45}$ haben die beiden ersten einen reellen Punkt gemein, ebenso die beiden letzten, weil in dem vollständigen Viereck 3456 sämmtliche Diagonalpunkte reell sind. Die Gattung III. enthält also 7 reelle, 16 imaginäre und 4 punktirte Gerade. ad IV. $s_{1}$ und $s_{2}, s_{3}$ und $s_{4}, s_{5}$ und $s_{6}$ sind conjugirt imaginär. Alle $G$ sind imaginär. Keiner der Kegelschnitte durch fünf der $s$ hat 
einen reellen Punkt, also sind auch alle $g$ imaginär. Den reellen Geraden $s_{1} s_{2}, s_{3} s_{4}, s_{5} s_{6}$ entsprechen drei reelle Gerade $l_{12}, l_{34}, l_{56}$. Jede der übrigen der 15 Verbindungsgeraden der $s$ hat, als in einer reellen Ebene liegend, einen reellen Punkt, der mit keinem der $s$ zusammenfällt, also ist die entsprechende Gerade auf $F_{3}$ punktirt. Die Gattung IV. enthält demnach 3 reelle, 12 imaginäre und 12 punktirte Gerade.

XI.

Aus den allgemeinen in dem Vorhergehenden behandelten Resultaten lassen sich durch Specialisiren einestheils des Flächenbündels, anderntheils der Ebene $E$, für deren Punkte die conjugirten Pole bestimmt werden, mannigfache Sätze ableiten, welche namentlich einen genaueren Einblick in die Beschaffenheit derjenigen Flächen dritten Grades gestatten, welche Doppelpunkte haben. Hier soll, um Weitläufigkeiten zu vermeiden, nur das Flächenbündel gewissen Bedingungen unterworfen werden, während die Lage der Ebene $E$ völlig willkürlich gelassen wird.

In VII. ist die nähere Bestimmung der Quadrupelcurve $C_{6}$ eines Flächenbündels unter der Voraussetzung gegeben worden, dass für keinen Punkt des Raumes die sämmtlichen Polarebenen in Bezug auf die Flächen zweiten Grades des Bündels zusammenfallen. Wird diese Bedingung aufgehoben und die ganz specielle Annahme gemacht, dass die sämmtlichen Flächen des Bündels ein gemeinsames Quadrupel haben, so zeigt es sich, dass die $C_{6}$ in die sechs Kanten dieses Quadrupels zerfällt. Werden dieselben durch eine Ebene $\boldsymbol{E}$ geschnitten, und wird die ihr entsprechende $F_{3}$ untersucht, so ergiebt sich zunächst, dass die Punkte $s$ die Ecken eines vollständigen Vierseits bilden, also viermal zu je dreien auf einer Geraden sich befinden, während die Geraden $G$ aus den Kanten des Quadrupels bestehen. Wird $s_{\lambda}$ von einer dieser Kanten ausgeschnitten, so ist $G_{\lambda}$ die dieser Kante gegenüberliegende. Die Geraden $G$ sind also nicht mehr windschief gegeneinander, sondern treffen sich zu je dreien in den Ecken eines Quadrupels, die demgemäss Doppelpunkte der $F_{3}$ sind.

Die Geraden $g$ entstehen aus den Kegelschnitten, welche je fünf der Grundpunkte enthalten. Sei $s(12345)$ ein solcher Kegelschnitt, so zerfällt derselbe in zwei Gerade, von denen jede drei der Punkte $s$ enthält, und einer solchen Geraden entsprechen auf $F_{3}$ einfach die den auf ihr liegenden 
Punkten $s$ zugehörigen Geraden $G$, demzufolge coincidiren in unserm Falle die $g$ und $G$. Was nun noch die Geraden $l$ betrifft, so ist zu unterscheiden, ob die entsprechende Gerade in $E$ zwei gegenüberliegende $s$ verbindet, oder aber zwei, die auf einer Seite des Vierseits der $s$ liegen. Im ersten Falle, der dreimal eintritt, erhält man eine neue Gerade der $\boldsymbol{F}_{3}$, im zweiten Falle aber treten nur die bereits bekannten Geraden $G$ auf. Um die Uebersicht zu erleichtern, nehmen wir an, dass von den sechs Punkten $s$ je auf einer Geraden liegen: 123, 156, 246, 345, so dass im Vierseit 123456 die Ecken 1 und 4, 2 und 5, 3 und 6 gegenüberliegende sind. Es befinden sich dann auf $F_{3}$ die Geraden:

$$
\begin{array}{ccc}
G_{1}=g_{4}=l_{23}=l_{56} ; \quad G_{2}=g_{5}=l_{13}=l_{46} ; \quad G_{3}=g_{6}=l_{12}=l_{45} \\
G_{4}=g_{1}=l_{26}=l_{35} ; \quad G_{5}=g_{2}=l_{34}=l_{16} ; \quad G_{6}=g_{3}=l_{15}=l_{24}
\end{array}
$$

Jede der sechs Kanten des Quadrupels ist also viermal als Gerade der $F_{3}$ zu zählen, jede der drei übrigen Geraden aber nur einmal, wie bereits anderweitig bekannt ist. - Da ein gemeinschaftliches Quadrupel zweier reellen Flächen zweiten Grades entweder vier reelle, oder zwei reelle und zwei conjugirt imaginäre, oder zweimal zwei conjugirt imaginäre Punkte hat, so ist klar, dass man leicht die auf die hier angegebene Weise zu erzeugenden Flächen dritten Grades mit vier Doppelpunkten nach der Realität dieser Doppelpunkte in drei Gattungen theilen kann, was hier nicht weiter ausgeführt werden soll.

Eine einfache Construction der Fläche dritten Grades mit vier Doppelpunkten beruht auf nachfolgenden Betrachtungen:

Vier nicht in einer und derselben Ebene gelegene Punkte $a, b, c, d$ sollen zu einem windschiefen Vierseit verbunden werden, in welchem $a$ und $c$, $b$ und $d$ gegenüberliegende Ecken, $d a=I$ und $b c=I I I, a b=I V$ und $c d=I I$ gegenüberliegende Seiten sind. Man kann nun in Bezug auf dieses Vierseit jeder Ebene $\boldsymbol{E}$ einen in ihr liegenden Punkt $p$ so zuordnen, dass, wenn 1, 2, 3, 4 die Durchschnitte von $E$ resp. mit $I, I I, I I I, I V$ sind, dann der gesuchte Punkt $p$ der Schnitt der Geraden 13 und 24 ist. Es ist umgekehrt leicht, zu dem Punkte $p$ die Ebene $E$ zu construiren, indem man einfach die beiden Geraden legt, welche von $p$ ausgehen und resp. $I$ und $I I I, I I$ und $I V$ schneiden, diese Geraden bestimmen die Ebene $E$ vollständig. Als Ausnahmefälle sind diejenigen zu betrachten, wenn $E$ eine Kante des Tetraeders abcd enthält, oder mit einer Seitenfläche desselben zusammenfällt, und wenn $p$ auf einer Kante liegt, oder in eine Ecke desselben fällt. 
Die besprochene Fläche wird nun erzeugt, indem man den Punkt $p$ für alle durch einen Punkt $\boldsymbol{P}$ gehenden Ebenen $\boldsymbol{E}$ construirt. Dies würde zunächst daraus folgen, dass wenn $E$ sich um eine Gerade $G$ dreht, dann der Punkt $p$ die Raumcurve dritten Grades durchläuft, welche der partielle Schnitt der Hyperboloide ist, die resp. durch die Geraden $I, I I I, G$ und $I I, I V, G$ bestimmt sind; anderntheils ergiebt sich das gesuchte Resultat als specieller Fall der dritten Steinerschen Erzeugungsart der Flächen dritten Grades. In der That, wenn $\boldsymbol{E}$ eine durch $\boldsymbol{P}$ gehende Ebene, $\boldsymbol{p}$ der ihr zugehörige Punkt ist, so lässt sich durch das windschiefe Vierseit III III IV und durch $p$ ein Hyperboloid legen, für welches $E$ eine Tangentialebene ist (denn $E$ schneidet dasselbe in zwei Geraden); demzufolge liegt $p$ auf dem Ort der Berührungspunkte aller von $P$ aus an das Hyperboloidenbüschel möglichen Tangentialebenen, und dieser Ort ist nach der angegebenen Steinerschen Erzeugungsart wirklich eine Fläche dritten Grades. Dass umgekehrt alle Punkte dieses Ortes auch Punkte $p$ sind, lässt sich leicht zeigen, ebenso, dass die Kanten des Tetraeders abcd ganz dem Orte von $p$ angehören, woraus folgt, dass derselbe eine Fläche vom dritten Grade mit den vier Doppelpunkten $a, b, c, d$ ist.

Die übrigen drei Geraden der Fläche bestimmen sich wie folgt: durch $P$ lege man die zugehörige Ebene $E$, so schneidet dieselbe das Vierseit IIIIIII $V$ in einem Viereck 1234, dessen Diagonaldreiseit ganz auf $F_{3}$ liegt. Eine Seite dieses Dreiseits ist nämlich die Polare des Punktes $P$ in Bezug auf das vorhin erwähnte Hyperboloidenbüschel und gehört deshalb der $F_{3}$ an, die beiden anderen aber enthalten den auf $F_{3}$ enthaltenen Punkt $\boldsymbol{P}$ und schneiden zudem je drei der Geraden der Fläche.

Schliesslich sei bemerkt, dass durch eine ganz ähnliche Construction die Steinersche Fläche dritter Klasse und vierten Grades hergestellt werden kann.

\section{XII.}

Wenn von den acht Schnittpunkten dreier Flächen zweiten Grades sieben auf einer irreductibeln Raumcurve dritten Grades $C_{3}$ liegen, so befindet sich der achte ebenfalls auf $C_{3}$, und das durch die acht Schnittpunkte bestimmte Flächenbündel zweiten Grades besteht aus den sämmtlichen Flächen zweiten Grades, welche durch $C_{3}$ gelegt werden können. Der Ort der Mittelpunkte aller im Bündel enthaltenen Kegel, mit anderen Worten die Quadrupelcurve des Bündels, reducirt sich demnach auf $C_{3}$. Conjugirte Punkte in Bezug auf 
das Bündel können leicht construirt werden, zu $p$ findet man den zugehörigen $p_{1}$, indem man durch $p$ die doppeltschneidende Gerade der $C_{3}$ zieht, welche mit $C_{3}$ die Punkte $s_{1}$ und $s_{2}$ gemein haben mag; der gesuchte Punkt $p_{1}$ ist dann der vierte harmonische, dem $p$ zugeordnete Punkt in Bezug auf $s_{1}$ und $s_{2}$. Zu jedem Punkte $p$ im Raum giebt es im Allgemeinen stets einen, aber auch nur einen Punkt $p_{1}$; die einzige Ausnahme bilden die Punkte der $C_{3}$ : jedem derselben entspricht die zugehörige Tangente der $C_{3}$.

Bewegt sich $p$ auf einer Geraden $g$, die $C_{3}$ nicht schneidet, so beschreibt $p_{1}$ eine Raumcurve dritten Grades $c_{3}$, welche, da $g$ und die Tangentenfläche von $C_{3}$ sich in vier Punkten treffen, mit $C_{3}$ nothwendig vier Punkte gemein hat. - Wenn aber $g$ mit $C_{3}$ einen Punkt $s$ gemein hat, so zerfällt die zugehörige $c_{3}$ in die Tangente, welche in $s$ an $C_{3}$ gelegt werden kann, und in ein Gebilde zweiten Grades. Zunächst ist klar, dass dasselbe auf dem Hyperboloide $\boldsymbol{H}_{2}$ liegt, welches durch $g$ und $C_{3}$ möglich ist, denn sämmtliche Erzeugende von $\boldsymbol{H}_{2}$, welche $g$ treffen, sind doppeltschneidende Geraden der $C_{3}$. Ferner bestimmt $g$ mit dem Kegel, welcher durch $C_{3}$ geht und $s$ zum Mittelpunkte hat, eine Polarebene, in der das gesuchte Gebilde ebenfalls enthalten sein muss, demzufolge ist es der Schnitt dieser Ebene mit $\boldsymbol{H}_{2}$, also ein Kegelschnitt, welcher $s$ enthält und $C_{3}$ ausser in $s$ noch in zwei anderen Punkten trifft. - Haben $g$ und $C_{3}$ zwei Punkte $s_{1}$ und $s_{2}$ gemein, so zerfällt $c_{3}$ in die Gerade $s_{1} s_{2}$ und die beiden Tangenten, welche in $s_{1}$ und $s_{2}$ an $C_{3}$ gelegt werden können. Ist schliesslich $g$ selbst eine Tangente der $C_{3}$, so besteht die ihr zugehörige $c_{3}$ aus dieser nämlichen Tangente dreifach gelegt.

Aus den bereits abgeleiteten allgemeineren Sätzen ergiebt sich, dass wenn $p$ eine Ebene durchläuft, die der $C_{3}$ gegenüber keine singuläre Lage hat, dann $p_{1}$ eine Fläche dritten Grades beschreib̆t. Dasselbe Resultat hätte man auch in nachfolgender Weise erhalten können: Seien $s_{1}, s_{2}, s_{3}$ die Durchschnittspunkte von $E$ mit $C_{3}$, dann findet man den Ort des Punktes $p_{1}$, indem man einfach den Ort der entsprechenden Gebilde für sämmtliche durch den Punkt $s_{1}$ gehende und in $E$ liegende Geraden bestimmt. Jeder solchen Geraden $g$ entspricht zunächst die Tangente in $s_{1}$ an $C_{3}$ und dann ein Kegelschnitt, der in der vorhin angegebenen Weise als Durchschnitt einer Ebene $e$ mit einem Hyperboloid $\boldsymbol{H}_{2}$ hergestellt werden kann. Dreht sich nun $g$ in $E$ um $s_{1}$, so beschreibt $e$ ein Ebenenbüschel und $H_{2}$ ein zu dem Ebenenbüschel projectivisches Hyperboloidenbüschel mit einer Grundcurve, die aus $C_{3}$ und der Geraden $s_{1} s_{2}$ besteht; der jeweilige Durchschnitt bewegt sich also, wie die 
zweite Steinersche Erzeugungsweise der Flächen dritten Grades lehrt, in einer Fläche dritten Grades $\boldsymbol{F}_{3}$.

Es ist jetzt zu untersuchen, ob auf dieser Fläche auch Geraden vorhanden seien und wie viele? - Da $s_{1}, s_{2}, s_{3}$ auf $C_{3}$ liegen, so gehören die Geraden $s_{2} s_{3}, s_{3} s_{1}, s_{1} s_{2}$ der Fläche $F_{3}$ an, ebenso liegen die in $s_{1}, s_{2}, s_{3}$ an $C_{3}$ möglichen Tangenten $t_{1}, t_{2}, t_{3}$ auf $F_{3}$, so dass also durch jeden der Punkte $s$ drei nicht in derselben Ebene liegende Geraden der $F_{3}$ gehen, d. h. diese hat die drei Punkte $s$ zu Doppelpunkten. Weitere Geraden der $F_{3}$ ergeben sich wie folgt: In Bezug auf den Kegel durch $F_{3}$ mit dem Mittelpunkte $s_{1}$ hat die Ebene $E$ eine bestimmte durch $s_{1}$ gehende Polgerade $\gamma_{1}$, die auch auf $F_{3}$ liegt, und der in $E$ ein ganz bestimmter Kegelschnitt entspricht, welcher die Punkte $s_{1}, s_{2}, s_{3}$ enthält; ähnlich findet man noch für $s_{2}$ und $s_{3}$ die Geraden $\gamma_{2}$ und $\gamma_{3}$. Es zeigt sich ferner, vermittelst eines Verfahrens, welches man bequem der zweiten Steinerschen Erzeugungsart der Flächen dritten Grades entnimmt, dass auf $F_{3}$ in den Ebenen $t_{1} \gamma_{1}, t_{2} \gamma_{2}, t_{3} \gamma_{3}$ noch je eine Gerade $\beta_{1}, \beta_{2}, \beta_{3}$ - liegt, deren Bild in $E$ eine resp. durch $s_{1}, s_{2}, s_{3}$ gehende Gerade ist, so dass also auf $F_{3}$ zwölf Geraden nachgewiesen sind, ausser welchen keine weiteren auf der Fläche enthalten sein können, wie sich sofort zeigen wird.

In Bezug auf die $C_{3}$ entspricht einer beliebigen Ebene $E$ eine Fläche dritten Grades $F_{3}$, einer zweiten Ebene $E^{\prime}$ gehört eine Fläche $F_{3}^{\prime}$ zu. Nun schneiden sich $F_{3}$ und $F_{3}^{\prime}$ in einer Raumcurve neunten Grades, welche aber zerfällt, und zwar in die $c_{3}$, welche der Schnittgeraden $g$ von $E$ und $E^{\prime}$ entspricht, und in eine Raumcurve sechsten Grades $C_{6}$. Diese $C_{6}$ kann nichts anderes sein, als die Quadrupelcurve $C_{3}$ doppelt gelegt, so dass also die sämmtlichen Flächen dritten Grades, welche durch Ebenen erzeugt werden, sich längs $C_{3}$ berühren, d. h. $C_{3}$ und eine andere, in jedem ihrer Punkte ihr unendlich nahe gelegene Raumcurve dritten Grades $C_{3}^{\prime}$ gemein haben.

Durch diese Bemerkung ist man in den Stand gesetzt, sofort nach VIII. die auf der hier betrachteten Fläche dritten Grades $F_{3}$ gelegenen Geraden abzuleiten. Denn seien $s_{4}, s_{5}, s_{6}$ die Durchschnittspunkte von $C_{3}^{\prime}$ mit $E$, welche resp. den Punkten $s_{1}, s_{2}, s_{3}$ unendlich nahe liegen, so dass immerhin die Geraden $s_{1} s_{4}, s_{2} s_{5}, s_{3} s_{6}$ eine bestimmte Richtung behalten, so bilden diese sechs Punkte zusammen die in VII. und VIII. näher definirten Grundpunkte $s$. Von den sechs Geraden $\boldsymbol{G}$ (um die dort eingeführte Bezeichnung beizubehalten) rücken also dreimal zwei zusammen:

$$
G_{1}=G_{4}, \quad G_{2}=G_{5}, \quad G_{3}=G_{6} .
$$


Ebenso leicht ist einzusehen, dass auch von den sechs Geraden $g$ je zwei zusammenfallen, also :

$$
g_{1}=g_{4}, \quad g_{2}=g_{5}, \quad g_{3}=g_{6} .
$$

Für die Geraden $l$ ergiebt sich die Tabelle:

$$
\begin{array}{ccc}
l_{23}=l_{26}=l_{53}=l_{56} ; & l_{31}=l_{61}=l_{34}=l_{64} ; & l_{12}=l_{15}=l_{42}=l_{45} ; \\
l_{14} ; & l_{25} ; & l_{36} .
\end{array}
$$

Jede der drei Geraden $s_{2} s_{3}, s_{3} s_{1}, s_{1} s_{2}$, wie sie vorhin genannt wurden, muss also viermal gezählt werden, jede der Geraden $t_{1}, t_{2}, t_{3} ; \gamma_{1}, \gamma_{2}, \gamma_{3}$ doppelt und jede der Geraden $\beta_{1}, \beta_{2}, \beta_{3}$ einfach, d. h. die Fläche dritten Grades mit drei Doppelpunkten, welche hier erzeugt worden ist, hat 3 quaternäre, 6 binäre und 3 unäre Geraden *).

\section{XIII.}

Wenn ein allgemeines Flächenbündel zweiten Grades fest bleibt, während die zu ihm in die bekannte Beziehung gesetzte Ebene nach und nach jede beliebige Lage annimmt, so entstehen unendlich viele Flächen dritten Grades, welche sämmtlich die Quadrupelcurve $C_{6}$ des Bündels enthalten, und von denen eine Einzelne erst dann bestimmt ist, wenn von ihr noch drei von einander unabhängige Punkte gegeben sind. Ein Complex von Flächen, welcher dreifach unendlich ist, heisst ein Netz, so dass also durch ein Flächenbündel zweiten Grades ein Netz von Flächen dritten Grades bestimmt ist.

In einem Flächennetz giebt es unendlich viele Flächen, welche einen Doppelpunkt haben, und der Ort derselben bildet eine Fläche, welche man als die Kernfläche des Netzes bezeichnen kann. Analytisch ist dieselbe leicht herzustellen, da man, um ihre Gleichung zu finden, nur die Jacobische Determinante für irgend vier von einander unabhängige Flächen des Netzes zu bilden und gleich Null zu setzen hat. Es ergiebt sich ohne Weiteres, dass für ein Netz von Flächen dritten Grades die Kernfläche vom achten Grade ist.

Für dasjenige Netz, welches durch ein Flächenbündel zweiten Grades bestimmt ist, lässt sich aber auf rein geometrischem Wege die Kernfläche mit

*) Sturm, a. a. O. No. 120. 
grösster Leichtigkeit bestimmen. Die Quadrupelcurve $C_{6}$ des Bündels hat nämlich die Eigenschaft, dass von irgend einem im Raume beliebig gewählten Punkte aus sieben geradlinige Strahlen gehen, welche dieselbe in zwei Punkten treffen, d. h. die $C_{6}$ hat sieben scheinbare Doppelpunkte. Wenn jetzt ein beliebiger Punkt $p$ des Raumes Doppelpunkt einer durch $C_{6}$ gehenden $F_{3}$ wäre, so müssten doch die sieben durch $\boldsymbol{p}$ gehenden doppeltschneidenden Geraden $\operatorname{der} C_{6} \operatorname{der} F_{3}$ angehören, was aber nicht möglich ist, weil von einem Doppelpunkte der $F_{3}$ aus höchstens sechs Geraden derselben gehen können. Soll aber $p$ wirklich ein Doppelpunkt sein, so müssen mindestens zwei der von ihm ausgehenden doppeltschneidenden Geraden der $C_{6}$ zusammenfallen, d. h. $p$ muss auf einer Geraden liegen, welche mit $C_{6}$ drei Punkte gemein hat. Alle diese Geraden aber bilden, wie am Schlusse von VllI. gezeigt wurde, eine geradlinige Fläche vom achten Grade, welche demnach die Kernfläche des Netzes ist.

Es knüpfen sich an diese Betrachtung die nachfolgenden Fragen, auf welche aber hier nicht eingegangen werden soll:

Wie sind die Flächen des vorliegenden Netzes beschaffen, welche zwei oder drei Doppelpunkte haben, resp. wie liegen diese Doppelpunkte? Wie viele Flächen mit drei Doppelpunkten sind in dem Netz enthalten?

Welches ist die Fläche, welche von den Ebenen umhüllt wird, die mit dem vorliegenden Flächenbündel zweiten Grades eine Fläche dritten Grades mit einem Doppelpunkt bestimmen? Welches ist die abwickelbare Fläche, welche von den Ebenen gebildet wird, die Flächen $F_{3}$ mit zwei Doppelpunkten bestimmen? Welche gegenseitige Beziehungen haben die Ebenen, welche Flächen $F_{3}$ mit drei Doppelpunkten ergeben?

\section{XIV.}

Ein merkwürdiges specielles Netz von Flächen dritten Grades wird durch vier gegen einander windschiefe und von einander unabhängige Geraden $I, I I, I I I, I V$ bestimmt. Jede $F_{3}$, auf welcher die vier Geraden liegen, enthält auch noch diejenigen beiden Geraden 1 und 2 (dieselben sind quadratisch, d. h. mit räumlichem Zirkel und Lineal zu finden), von denen jede die vier ursprünglich gegebenen schneidet, und so hat man ein Netz von Flächen dritten Grades mit einer Grundcurve sechsten Grades, die aus sechs Geraden besteht. Eine einzelne Fläche des Netzes wird erst bestimmt, wenn von ihr 
noch drei weitere von einander unabhängige Punkte gegeben sind, denn die vier Geraden legen der $F_{3}$ nur 16 Bedingungen auf, während bekanntlich zu deren Bestimmung 19 Bedingungen erforderlich sind.

Es soll jetzt diejenige der Flächen des Netzes bestimmt werden, welche durch die Punkte $p_{1}, p_{2}, p_{3}$ geht. Die Ebene $e$ dieser Punkte schneide die Geraden $I, I I, I I I, I V$ resp. in den Punkten $s_{I}, s_{I I}, s_{I I I}, s_{I V}$ und die Geraden 1 und 2 resp. in $s_{1}$ und $s_{2}$, so geht durch die neun Punkte $s$ und $p$ eine ebene Curve dritten Grades $C_{3}$, welche ganz der gesuchten Fläche $F_{3}$ angehört, und zwar ist es möglich, beliebig viele Punkte dieser Curve mittelst des Lineals allein zu construiren. - Durch die drei Geraden $I I, I I I, I V$ ist ein Hyperboloid $\boldsymbol{H}_{2}$ bestimmt, welches $\boldsymbol{F}_{3}$ in drei weitern Geraden trifft, von denen zwei die Geraden 1 und 2 sind. Die dritte ergiebt sich, indem man denjenigen Durchschnittspunkt des in $e$ gelegenen Kegelschnitts von $\boldsymbol{H}_{2}$ mit $C_{3}$ sucht, welcher keiner der Punkte $s$ ist; durch diesen auf linealem Wege construirbaren Punkt geht dann eine Gerade $g_{1}$, welche II, III und IV gleichzeitig schneidet, und welche denzufolge der $F_{3}$ angehört. In ähnlicher Weise findet man auf den Hyperboloiden (III IV I ), (IV I II), (I II III) noch drei neue Geraden $g_{2}, g_{3}, g_{4}$ der $F_{3}$, so dass also von derselben bereits $10 \mathrm{Ge}-$ raden bekannt sind. Von diesen bilden 1, 2, $g_{1}, g_{2}, g_{3}, g_{4}$ die Hälfte einer Schläflischen Doppelsechs. Es zeigt sich nun, dass die Construction der 27 Geraden der $F_{3}$ in diesem Falle nur die Lösung einer quadratischen Gleichung erfordert, während die Construction der 27 Geraden einer durch 19 ihrer Punkte gegebenen $F_{3}$ auf eine Gleichung sechsten Grades führt. Es ist übrigens klar, dass die Construction der $F_{3}$ unter den hier angenommenen speciellen Bedingungen sich auch leicht vermittelst der Grassmannschen Erzeugungsart ausführen lässt, da ja die projectivische Beziehung dreier die Fläche erzeugenden Ebenenbündel, deren Mittelpunkte die Punkte $p$ sind, unmittelbar gegeben ist.

Man kann für das betrachtete Netz die Kernfläche sofort bestimmen. Wäre ein beliebig im Raume gelegener Punkt Doppelpunkt einer Fläche des Netzes, so könnte man auf dieser sofort sieben durch den Punkt $p$ gehende Geraden angeben, von denen sechs die Schnittlinien der Ebenen sind, welche man durch $p$ und je eine der Geraden $I, I I, I I I, I V$ legen kann, während die siebente, von $p$ ausgehend, noch die Geraden 1 und 2 trifft, was ein Widerspruch ist. Dieser Widerspruch wird gehoben - und nur dann kann $p$ ein Knotenpunkt der $F_{3}$ sein, wenn $p$ auf einem der Hyperboloide liegt, welche 
durch irgend drei der Geraden $I, I I, I I I, I V$ gelegt werden können, d. h. die Kernfläche des Netzes besteht aus vier Hyperboloiden.

Anmerkung. Die vorliegende Abhandlung war im Manuscripte bereits vollkommen beendigt, als der erste Theil der Preisschrift des Herrn Cremona über Flächen dritten Grades erschien (Bd. 68 dieses Journals, Heft 1). Dieselbe konnte demnach nicht benutzt und im Literaturnachweise aufgeführt werden, obschon einzelne Sätze, welche sie enthält, auch in den hier gegebenen Entwicklungen sich finden. -

Zürich, den 9. Jan. 1868. 